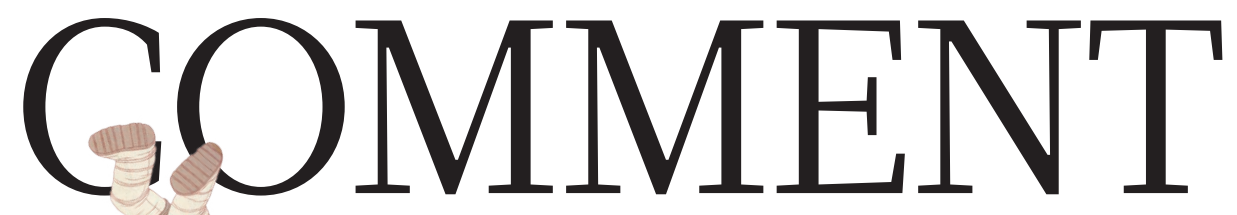

SOCIAL SCIENCE Take a broader, longer view of the Anthropocene $\mathbf{p . 1 9 2}$
CHILDREN The bedtime stories that set Nature's editors on the road to science $\mathbf{p . 1 9 4}$
CONSERVATION Halt catastrophic extinctions in Brazil's Cerrado $\mathbf{p} \mathbf{1 9 9}$
COMMUNITY Technicians need career paths, metrics and rewards p.199

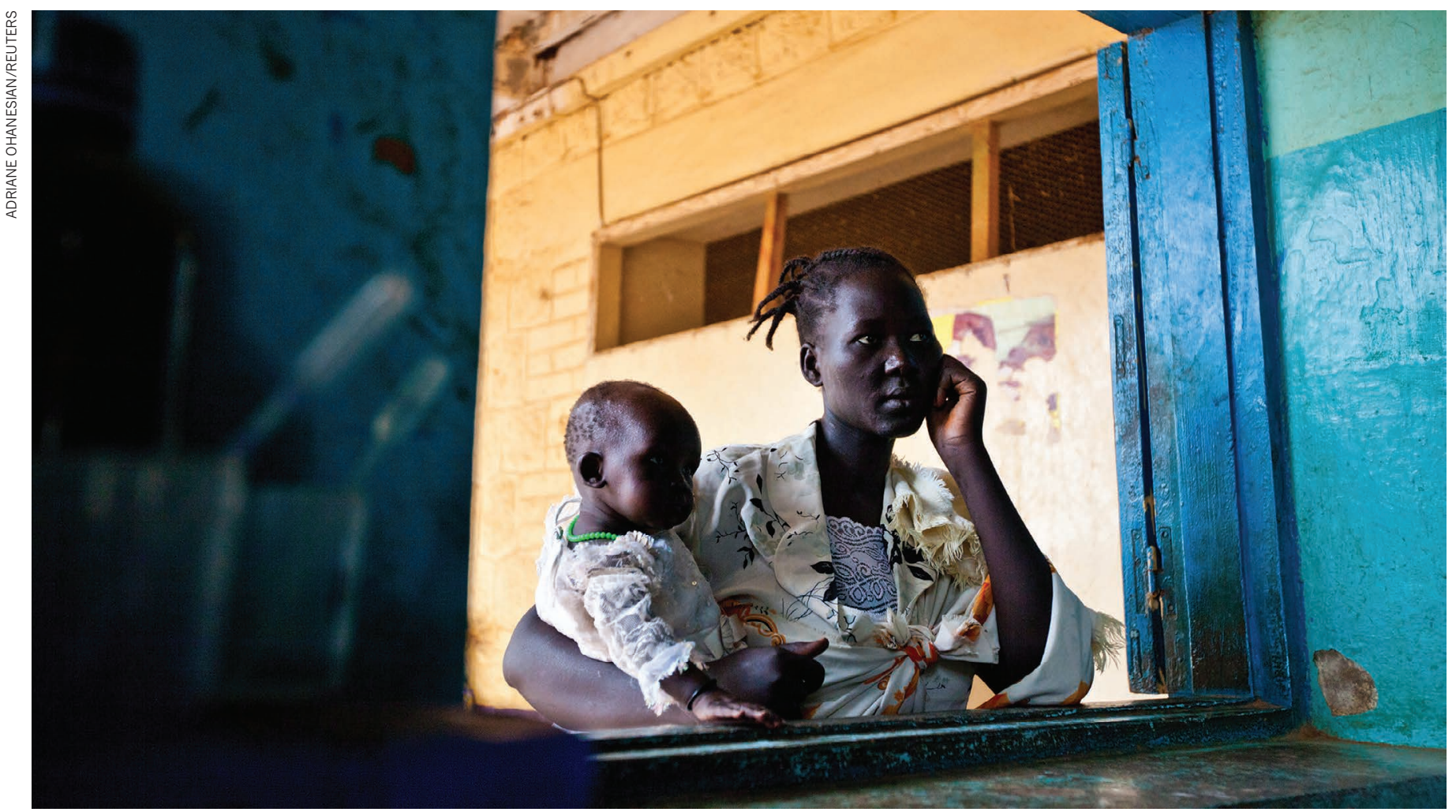

A mother and her child at a hospital in South Sudan, one country where precise public-health data are lacking.

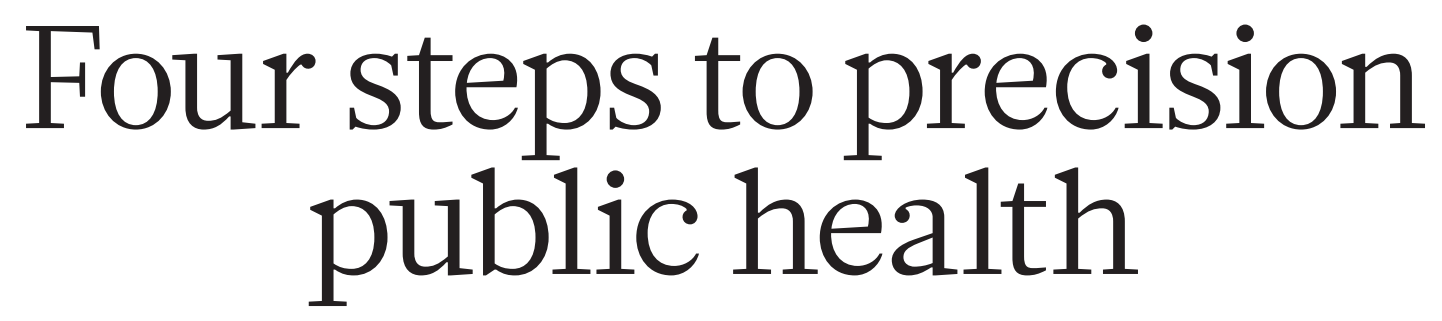

Better surveillance data and analyses are urgently needed to control disease in the developing world, argue Scott F. Dowell, David Blazes and Susan Desmond-Hellmann.

$\mathrm{W}$

hen domestic transmission of Zika virus was confirmed in the United States in July 2016, the entire country was not declared at risk nor even the entire state of Florida. Instead, precise surveillance defined two at-risk areas of Miami-Dade County, neighbourhoods measuring just 2.6 and 3.9 square kilometres. Travel advisories and mosquito control focused on those regions. Six weeks later, ongoing surveillance convinced officials to lift restrictions in one area and expand the other.

By contrast, a campaign against yellow fever launched this year in sub-Saharan Africa defines risk at the level of entire nations, often hundreds of thousands of square kilometres. More granular assessments have been deemed too complex.

The use of data to guide interventions that benefit populations more efficiently is a strategy we call precision public health. It requires robust primary surveillance data, rapid application of sophisticated analytics to track the geographical distribution of disease, and the capacity to act on such information ${ }^{1}$.

The availability and use of precise data is becoming the norm in wealthy countries. But large swathes of the developing world are not reaping its advantages. In Guinea, it took months to assemble enough data to clearly identify the start of the largest Ebola outbreak in history. This should take $>$ 


\section{STRATEGIC DEFENCE}

Ninety per cent of the disease burden can be addressed by focusing on just $14 \%$ of the total area in which the mosquito Aedes aegypti transmits chikungunya, dengue, yellow fever and Zika.

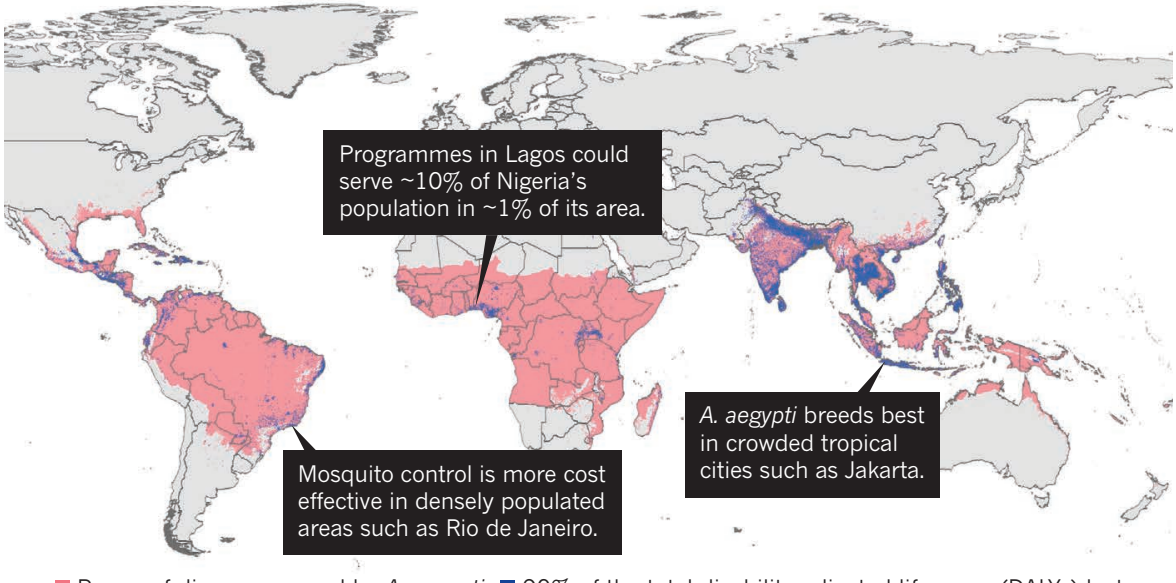

Range of diseases spread by A. aegypti $\square 90 \%$ of the total disability adjusted life years (DALYs) lost

days. Sub-Saharan Africa has the highest rates of childhood mortality in the world; it is also where we know the least about causes of death.

We at the Bill \& Melinda Gates Foundation in Seattle, Washington, are committed to seeing data used equally in the developing world. The United Nations' Sustainable Development Goals, launched in September 2015, will require ever more accurate and timely data to track and achieve progress. We are calling on government leaders and global health workers to join us in revamping surveillance to make precision public health a reality.

\section{DATA TRENDING}

The value of precise disease tracking was baked into epidemiology from the start. In 1854, John Snow famously located cholera cases in London. His mapping of the spread of infection through contaminated water dealt a blow to the idea that the disease was caused by bad air. These days, people and pathogens move across the globe swiftly and in great numbers. In 2009, the H1N1 'swine flu' influenza virus took just 35 days to spread from Mexico and the United States to China, South Korea and 12 other countries.

Fortunately, data are also more available than ever before. The Global Polio Eradication Initiative routinely sequences all poliovirus isolates collected from people diagnosed with the disease. The presence of viruses with relatively large ( $>1.5 \%)$ sequence differences from other isolates can indicate where surveillance and vaccination efforts have failed, and suggest how to redirect efforts to reduce undetected transmission ${ }^{2}$.

Large-scale collections of the world's health data are catalogued (such as by the Global Health Data Exchange: http://ghdx. healthdata.org) and have been used to compile an inventory of the global burden of disease. The 2015 report from the Institute for Health Metrics and Evaluation in Seattle enlisted more than 1,800 collaborators who analysed more than 14,000 data sets to estimate mortality for 249 causes in 195 countries across 35 years ${ }^{3}$.

The public-health community is sharing more data faster; expectations are higher than ever that data will be available from clinical trials and from disease surveillance. In the past two years, the US National Institutes of Health, the Wellcome Trust in London and the Gates Foundation have all instituted open data policies for their grant recipients, and leading journals have declared that sharing data during disease emergencies will not impede later publication (see go.nature.com/2gcwh4a).

Meanwhile, improved analysis, data visualization and machine learning have expanded our ability to use dis-

parate data sources "Data quality to decide what to do. is of ten too A study published poor to drive last year ${ }^{4}$ used precise decisions." geospatial modelling to infer that insecticide-treated bed nets were the single most influential intervention in the rapid decline of malaria.

However, in many parts of the developing world, there are still hurdles to the collection, analysis and use of more precise public-health data. Work towards malaria elimination in South Africa, for example, has depended largely on paper reporting forms, which are collected and entered manually each week by dozens of subdistricts, and eventually analysed at the province level. This process would be much faster if field workers filed reports from mobile phones ${ }^{5}$.

\section{SPEED, ACCURACY, EQUITY}

Precision public health can save lives. Meningococcal disease in West Africa can go from scattered cases to devastating outbreaks in weeks. Since 1997, regular surveillance has triggered mass vaccination campaigns when clusters of disease surpass set thresholds. A 2015 analysis $^{6}$ of surveil lance data in Niger found that monitoring at the level of 'health areas' (median population of 14,440), rather than at the district level (median population of 295,200 ), would increase the signals detected and reduce the delay in response. Accelerating effective vaccination by just two weeks would interrupt any outbreak more quickly, halving the number of cases. However, the analysis also showed that such outcomes would require more-frequent data analysis and decentralized vaccine storage.

More-precise disease surveillance can also illuminate causes of disease and spark opportunities for prevention. In the pilot phase of a new Gates Foundation surveillance programme, investigators in Soweto in South Africa found evidence of group B streptococci in the tissues of stillbirths (see 'Disease surveillance'). Women who have experienced such losses also have a sevenfold higher risk of recurrence in their next pregnancy. Penicillin can prevent group B streptococcal infections in newborns, and prescribing the antibiotic to women with risky pregnancies is feasible and affordable for poor communities such as Soweto - but only if health workers know how to target treatment to the fraction of patients who need it. Thus, improved surveillance and analysis allows an impoverished population to receive an intervention that is standard practice in wealthy countries, but which is not used in much of the developing world.

Geographic precision also means that public-health resources are used more efficiently, so more people receive interventions. For example, the Aedes aegypti mosquitoes that transmit dengue, Zika and chikungunya viruses do not spread these infections if they carry Wolbachia, a benign symbiotic bacterium ${ }^{7}$. Wolbachiacarrying mosquitoes can be distributed to displace wild-type A. aegypti and reduce transmission of these viral diseases, but the wide global prevalence of these arboviruses makes this approach daunting and potentially costly ${ }^{8}$

Fortunately, disease-distribution information and geospatial modelling has pinpointed the areas that have $90 \%$ of the predicted disease burden (see 'Strategic defence'). This reduces the area for intervention from some 50 million square kilometres to 7 million square kilometres, and promises to make Wolbachia-based mosquito control feasible and affordable for many more countries. Demonstration projects in Medellin in Colombia and Rio de Janeiro in Brazil began in late 2016.

Still, we must try harder. Despite progress, data quality is often too poor to drive decisions. Data sets are limited, testing is unreliable and outcomes from vast regions 
are unavailable. In 2013, errors in Google Flu Trends, an open flu-surveillance tool, showed that big-data analytics alone can supplement but not replace conventional surveillance ${ }^{9}$. Furthermore, the cause of most deaths is never adequately identified — in 2008 , only $4 \%$ of childhood deaths worldwide occurred in countries that have an adequate medical certification procedure.

\section{ACHIEVING PRECISION}

Four concrete steps are necessary for precision public health to become regularly available in the developing world.

Register births and deaths. It is hard to know whether a national deworming programme for children in Sierra Leone or a vaccination programme for whooping cough (pertussis) in Nigeria is reducing mortality when less than $4 \%$ of deaths are registered.

These basic demographic data are necessary for rational public-health decisions. To achieve the Sustainable Development Goals, the World Bank Group and its partners should expand support for systems to sample representative areas for births and deaths in all high-mortality countries by 2020 , as a step towards full civil registration and vital statistics in all countries by 2030 . Our estimates put this cost at less than US\$1 million per year for countries with 30 million people or fewer.

Track disease. Careful surveillance can guide public health in a country and track disease outbreaks that could spread beyond borders. This requires infrastructure and systems to collect and analyse data,

laboratories to confirm diagnoses, and adequately trained personnel. All 194 member states of the World Health Organization (WHO) should fulfil their obligations under the International Health Regulations to achieve a minimum core capacity for surveillance. When countries are too poor for this, G20 countries should help. The estimated cost of pandemic preparedness is about $\$ 4.5$ billion - less than $0.005 \%$ of the world's gross domestic product.

At the 2017 World Health Assembly in May, these rich countries should commit to implementing concrete support for at least 76 of the neediest ones. Countries should submit to objective, published, external evaluations of their surveillance capacity and pan-

"The cause of most deaths is never adequately identified." demic preparedness; self-reports of progress are not acceptable. The Joint External Evaluations, led by the WHO and building on targets developed under the Global Health Security Agenda, should guide this process.

Incorporate laboratory analyses. The cause of a child's death cannot be adequately determined by interviews with relatives, especially months after death. Where feasible, tissue sampling and laboratory diagnosis should supplement or replace these 'verbal autopsies. Our initial experience is that most relatives consent readily, because families want to know why their child died. Autopsybased mortality surveillance is expensive more than \$5 million per country per year

\section{DISEASE SURVEILLANCE}

\section{Why investment is key}

The Bill \& Melinda Gates Foundation did not initially focus on disease surveillance; this is conventionally a government function. Yet it became obvious that the lack of data prevented us from modelling disease or designing effective vaccine trials.

In 2014, we established a team focused on surveillance and epidemiology. Its first task was tracking childhood mortality using a technology called minimally invasive tissue sampling. This provides more precise information than interviews with relatives about deceased children's symptoms.

These efforts have grown into a network of sites across Africa and South Asia called Child Health and Mortality Prevention Surveillance (CHAMPS). The sites are geographically limited, with well-defined populations of 150,000 to 300,000 people. Active outreach by community health workers helps to identify pregnancies and deaths of children under five, and to obtain tissue samples for definitive identification of the causes. CHAMPS will now be integrated with efforts to track mortality countrywide in Mozambique and elsewhere.

Pathology findings from CHAMPS sites will be combined with ongoing programmes from the World Bank, UNICEF, the World Health Organization, Canada, the US Agency for International Development and the US Centers for Disease Control and Prevention to collect vital national statistics. The Gates foundation plans additional investments in these programmes. This information will be fed into the data sets accessed by the Institute for Health Metrics and Evaluation in Seattle, Washington, and others.

This will expand mapping capacity and improve estimates of the global distribution of disease and the impacts of public-health interventions.
- but can strengthen broader surveillance efforts. By pairing just a small number of physical autopsies with verbal autopsies on the same deaths, the much larger number of verbal autopsies can be calibrated to more accurately reflect the true distribution of mortality causes.

Train more people. Public-health personnel at national and local levels must be educated in the fundamentals of epidemiology, empowered to use local information to set strategies, and equipped to translate those decisions into action.

Relevant resources exist: field epidemiology and laboratory training programmes such as FETP, supported by the US Centers for Disease Control and Prevention, are highly effective. Software such as Epi Info 7 provides basic analysis for outbreak management, and other open-source software products assist with disease surveillance, such as the Suite for Automated Global Electronic bioSurveillance (SAGES) from Johns Hopkins University. However, these are not deployed in many places where they are desperately needed.

Training costs are not high - we estimate that salaries would be needed for perhaps a dozen doctoral-level personnel per country, but even wealthy countries struggle to maintain budgets for public-health workers when there is no crisis to put pressure on politicians. The WHO and other players must keep the pressure on.

Frontline workers should not find themselves frustrated by global programmes that fail to take into account data on local circumstances. Wherever they live - in a village, city or country, in the global south or north - people have the right to public-health decisions that are based on the best data and science possible, that minimize risk and cost, and maximize health in their communities.

Scott F. Dowell is deputy director for surveillance and epidemiology; David Blazes is senior programme officer for surveillance and epidemiology; and Susan Desmond-Hellmann is chief executive at the Bill \& Melinda Gates Foundation, Seattle, Washington, USA.

e-mail:scott.dowell@gatesfoundation.org

1. Desmond-Hellmann, S. Science 353, 731 (2016).

2. Wassilak, S. G. F. et al. J. Infect. Dis. 210 (Suppl. 1) S5-S15 (2014)

3. GBD 2015 Mortality and Causes of Death Collaborators. Lancet 388, 1459-1544 (2016).

4. Bhatt, S. et al. Nature 526, 207-211 (2015).

5. Quan, V., Hulth, A., Kok, G. \& Blumberg, L. Malaria J. 13, 151 (2014).

6. Maïnassara, H. B et al. Emerg Infect Dis. 21 , 1322-1329 (2015)

7. Ferguson, N. M. et al. Sci. Transl. Med. 7, 279 ra37 (2015).

8. Kraemer, M. U. G. et al. eLife 4, e08347 (2015).

9. Olson, D. R., Konty, K. J., Paladini, M., Viboud, C. \& Simonsen, L. PLoS Comput. Biol. 9, e1003256 (2013). 